\title{
Conformabilidad de láminas de acero AISI/SAE 1006 utilizadas para estampado/embutición en el Valle de Aburrá
}

\author{
Formability of AISI/SAE 1006 steel sheets used for stamping/drawing in the Valley of
}

\author{
Aburrá \\ Jesús David Mazuera Robledo ${ }^{1}$, Jorge Iván Gómez Gómez ${ }^{2}$, Elena Isabel Mercado Chavarría ${ }^{3}$. \\ Departamento Ingeniería Mecánica, Universidad Nacional de Colombia Sede Medellín, Medellín, Colombia \\ ${ }^{3}$ Departamento Ingeniería Industrial, Universidad de Antioquia, Medellín, Colombia \\ jdmazuereunal. edu.co \\ jigomezgeunal.edu.co \\ jazabela86@hotmail.com
}

\begin{abstract}
Resumen- Se caracterizaron láminas de acero AISI/SAE 1006 provenientes de tres fabricantes diferentes para buscar la causa de la aparición de defectos como rasgaduras reportados por los industriales del Valle de Aburrá cuando cambian de proveedor de las láminas. En la caracterización se determinaron la composición química, resistencia a la cedencia, resistencia a la tracción, coeficiente de anisotropía (r) y razón de anisotropía plástica normal (rm). Adicionalmente, a partir de los datos obtenidos en la caracterización, se realizaron simulaciones por elementos finitos para corroborar la problemática reportada. Finalmente, se llevaron a cabo visitas a comercializadores y consumidores de lámina para estampado/embutición para determinar su grado de conocimiento de los parámetros que determinan la conformabilidad de dichos materiales. Las simulaciones corroboraron la aparición de defectos en dos de los materiales analizados y se encontró que la problemática se debe mayormente al desconocimiento tanto de comercializadores como de consumidores de lámina frente a los parámetros que determinan su conformabilidad; lo que conlleva a que la adquisición de los materiales se haga con base en especificaciones para aceros de uso general o aceros estructurales cuyos criterios de selección no brindan información relevante para el proceso de manufactura para el que son adquiridos.
\end{abstract}

Palabras clave- acero AISI/SAE 1006, coeficiente de anisotropía, conformabilidad, exponente de endurecimiento por deformación.

Abstract- AISI/SAE 1006 steel sheets from three different suppliers were characterized to determine the cause of defects such as tearing that, according to reports from industries located in the Valley of Aburra, appeared on stamped/drawn pieces when the steel supplier was changed. The characterization relied on chemical composition, metallographic analysis, yield strength, tensile strength, anisotropy coefficient (r) and the normal plastic anisotropy ratio $(\mathrm{rm})$. In addition, based on the results obtained during characterization finite element simulations were conducted to verify the reported issue. Finally, visits to supplier and consumers of steel sheet used for drawing and stamping

Fecha de Recepción: 28 de Mayo de 2014

Fecha de Aceptación: 25 de Marzo de 2015 operations were carried out to establish their level of knowledge related to parameters that determine the behavior of steel sheets during the forming processes. Results of simulations confirmed the appearing defects and it was found that their main cause was the lack of knowledge of Colombian suppliers and consumers about parameters that determine the plastic behavior of steel sheets which led to make the steel purchase process based on specifications for general use steels that do not provide useful information about the manufacture process the sheets were bought for.

Key Word - AISI/SAE 1006 steel, anisotropy coefficient, formability, strain hardening exponent.

\section{INTRODUCCIÓN}

El acero AISI/SAE 1006 es un acero simple al carbono de uso general que se produce en diferentes formas como barras, láminas y chapa calibrada (lámina delgada) $[1,2]$. Esta última es uno de los materiales más usados por diferentes industrias del Valle de Aburrá para la fabricación de piezas mediante operaciones de estampado y embutición para sectores como "línea blanca", autopartes, componentes eléctricos, etc. Los bajos volúmenes de producción que se manejan en el país en dichos sectores (en comparación con otros países consumidores de lámina), obligan a que se cambie de manera constante los proveedores de este tipo de lámina dependiendo de las fluctuaciones del mercado mundial de acero dado que en época de escasez se recorta el suministro hacia consumidores pequeños como Colombia. Por lo anterior, es común que al país lleguen láminas procedentes de Venezuela, Brasil, Corea, Japón, etc, lo que conduce, según lo reportan los industriales localizados en el Valle de Aburrá, a la aparición de defectos (principalmente rasgaduras) en las piezas estampadas o embutidas que obligan a realizar ajustes de la maquinaria y el proceso cada vez que se cambia el 
proveedor de la lámina conllevando en algunos casos a que la materia prima deba ser descartada o, en el mejor caso, destinada a la fabricación de productos que requieran operaciones de deformación menos exigentes como doblados o rolados.

Es pertinente mencionar que la problemática mencionada puede estarse presentando a nivel nacional y extenderse a otros tipos de aceros destinados a operaciones de estampado y embutición. Sin embargo, el presente trabajo se enfocó en el análisis de conformabilidad de láminas de acero ASIS/SAE 1006 que se comercializan en el Valle de Aburrá. Para ello se caracterizaron láminas de acero AISI/SAE 1006 provenientes de tres fabricantes diferentes y se analizó su conformabilidad con la ayuda de simulación por elementos finitos para determinar la causa de la problemática antes mencionada. De igual manera, se realizaron entrevistas a comercializadores y consumidores de lámina de acero AISI/SAE 1006 destinada a embutición para determinar si el flujo de información en el proceso de comercialización y compra del acero pudiera tener alguna influencia en el problema reportado por los industriales al cambiar la procedencia de la lámina.

\section{FUNDAMENTACIÓN TEÓRICA}

\section{A. Parámetros usados en la definición la conformabilidad de la lámina de acero de bajo carbono}

Cuando se trata de analizar problemas de conformabilidad en metales, la información que se obtiene de una curva esfuerzo deformación de ingeniería, que es la que usualmente entregan los fabricantes de láminas de acero comercializadas en Colombia (y en particular en el Valle de Aburrá), resulta insuficiente debido a que los esfuerzos y las deformaciones en ella calculadas se obtienen con base en las dimensiones iniciales de la probeta; y por tanto, no pueden dar cuenta de las respuesta instantánea del material cuando se deforma plásticamente. Por lo anterior, para inferir el comportamiento de una lámina metálica delgada en un proceso de conformado, es usual en los países industrializados, partir de la curva esfuerzo verdadero $(\sigma \mathrm{T})$ - deformación verdadera $(\varepsilon T)$ cuya región de deformación plástica (antes del punto de máxima carga) se puede aproximar con la expresión mostrada en la ecuación (1); Allí, k es el coeficiente de endurecimiento por deformación y $\mathrm{n}$ se conoce como el exponente de endurecimiento por deformación [3-7], los cuales se determinan siguiendo el procedimiento estándar ASTM E646 [7].

$$
\sigma_{T}=k\left(\varepsilon_{T}\right)^{n}
$$

Por otro lado, las chapas de acero laminadas en frío presentan una microestructura con granos alargados en la dirección de laminación que les confieren anisotropía (se puede decir que la mayoría de estas láminas son ortotrópicas) en las propiedades mecánicas. El grado de ansiotropía induce cambios en el comportamiento de una lámina cuando se someta a operaciones de estampado/embutido, por lo que debe evaluarse dicho comportamiento. Tal evaluación se realiza haciendo uso de una cantidad denominada parámetro de Lankford, coeficiente de anisotropía o razón de deformación plástica [3-6,8], que se representa por la letra r y está definido por la ecuación (2).

$$
r=\varepsilon_{T 2} / \mathcal{E}_{T 3}
$$

Donde $\varepsilon$ T2 y $\varepsilon$ T3 son las deformaciones en el ancho y el espesor de la probeta respectivamente (El método detallado para la obtención de este parámetro puede revisarse en la norma ASTM E517 [8]). Para el análisis de problemas de conformabilidad es una práctica común medir el valor de $\mathrm{r}$ a $0^{\circ}, 45^{\circ}$ y $90^{\circ}$ con respecto a la dirección de laminación del material (los valores así obtenidos se nombran como r0, r45 y r90) y determinar la razón de anisotropía plástica normal, rm, definida por la ecuación (3).

$$
r_{m}=\left(r_{0}+2 r_{45}+r_{90}\right) / 4
$$

Las normas SAE J2329 y la ASTM 1008 [9,10], son estándares usados en EUA, a la hora de precisar los parámetros que deben caracterizar una chapa de acero destinada a embutición; dichas normas, son también de amplia aceptación a nivel internacional. En ellas, las láminas de acero al carbono de bajo carbono son clasificadas según: composición química, resistencia a la cedencia, resistencia a la tracción, exponente endurecimiento por deformación plástica y razón de anisotropía plástica normal. Es importante mencionar que existen otros parámetros que definen el comportamiento de láminas metálicas durante procesos de conformado tales como la curva límite de conformado (FLC) y el coeficiente de sensibilidad a la velocidad de deformación (m) que no fueron analizados en este trabajo, ya que no son requeridos por los estándares SAE J2329 y ASTME1008 $[9,10]$.

\section{B. Simulación inversa}

El método inverso (también conocido como "one step" en inglés) de simulación por elementos finitos para procesos de estampado/embutición de lámina metálica delgada, es una formulación simplificada en la que se desprecian factores como la historia de las fuerzas de contacto y los coeficientes de fricción entre lámina y el herramental. También se simplifican los modelos asumiendo que cada elemento finito, partiendo de un blanco plano, llega a su posición final (en la forma definitiva de la pieza conformada) siguiendo una trayectoria recta y en un solo paso (de allí su nombre en inglés de "one step"), como se muestra esquemáticamente en la figura 1. De esta manera se logra reducir sustancialmente el tiempo de simulación para evaluar de manera rápida la viabilidad de estampado de una pieza en su etapa de diseño conceptual o de detalle, partiendo de la geometría de la pieza 
estampada [11,12]. Este método de simulación requiere como datos de entrada la geometría de la pieza conformada, la fuerza aplicada en el prensachapa (cuando se usa éste) y parámetros del material tales como resistencia a la cedencia, resistencia a la tracción, módulo elástico, relación de anisotropía (medida a $0^{\circ}, 45^{\circ}$ y $90^{\circ}$ con respecto a la dirección de laminación del material), coeficiente de endurecimiento por deformación, exponente de endurecimiento por deformación, razón de Poisson y curva límite de conformado (FLC).

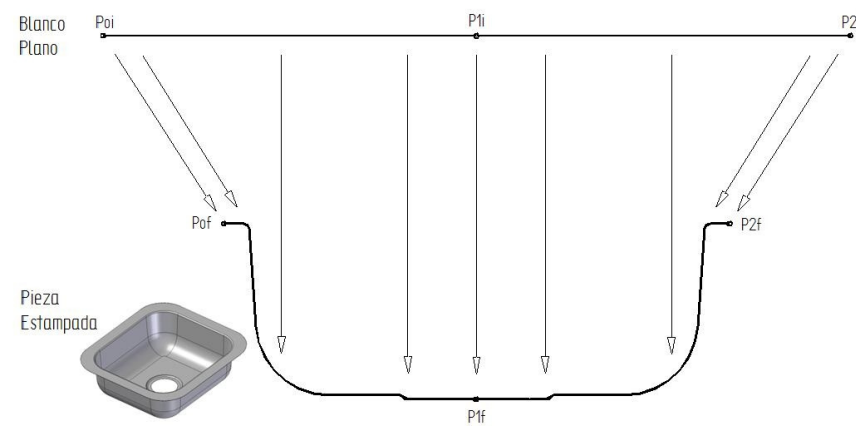

Figura 1. Representación esquemática del método de simulación inverso, se muestra la sección transversal del blanco plano y la pieza estampada

\section{PROCEDIMIENTO EXPERIMENTAL}

\section{A. Materiales}

Se utilizaron láminas de acero AISI/SAE 1006 calibre 24 (0,6 $\mathrm{mm}$ de espesor), provenientes de tres proveedores diferentes en estado de suministro. Por consideraciones de confidencialidad las láminas fueron identificadas de manera genérica como acero 1 , acero 2 y acero 3 . Este material generalmente es solicitado, comercializado y recibido como lámina "cold rolled" (laminada en frío).

\section{B. Caracterización de materiales}

La caracterización de los materiales involucrados en el presente estudio se llevó a cabo mediante mediciones de composición química, ensayos mecánicos de tracción y análisis metalográfico. La preparación de las muestras para el análisis metalográfico se llevó a cabo mediante la técnica tradicional de pulido hasta superficie espejo y posterior ataque con Nital al 0.5\%, Las mediciones de composición química se realizaron mediante espectrometría de emisión óptica (GDS).

Para cada uno de los materiales estudiados se cortaron, usando chorro de agua, probetas de tracción orientadas a $0^{\circ}, 45^{\circ}$ y $90^{\circ}$ con respecto a la dirección de laminación de la lámina. Estas probetas fueron utilizadas para determinar la razón de deformación plástica (r) siguiendo el procedimiento estándar ASTM E517 [8]. Adicionalmente se cortó una probeta orientada en la dirección de laminación para medir la resistencia a la cedencia y la resistencia a la tracción de acuerdo con el estándar ASTM A370 [13]. Todos los ensayos de tracción se llevaron a cabo a una velocidad de $25 \mathrm{~mm} / \mathrm{min}$ usando la probeta mostrada en la figura 2 (cuya longitud calibrada es de $50 \mathrm{~mm}$ ) para satisfacer los requerimientos de los estándares ASTM E517 y ASTM A370.

Debido a que no se contaba con el montaje experimental adecuado (en particular con un extensómetro) requerido por el estándar ASTM E646 [7], no fue posible realizar la medición del exponente de endurecimiento por deformación (n) y el coeficiente de endurecimiento por deformación (k).

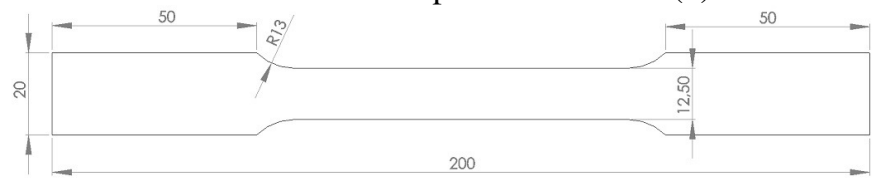

Figura 2. Geometría de las probetas usadas para los ensayos de tracción

\section{Simulación inversa}

Para corroborar la problemática reportada por los industriales al cambiar el proveedor de la lámina, se llevó a cabo la simulación de una operación de embutición profunda con una geometría simple (ver Figura 1) usando el método inverso en el paquete Altair HyperForm. Los materiales utilizados para la simulación se seleccionaron de la base de datos de HyperForm a partir de los datos obtenidos en la caracterización de materiales, con los que se determinó la clasificación de cada uno de acuerdo con el estándar SAE J2329 [10]. En todos los casos se usó la misma geometría de pieza conformada y una fuerza de 25 toneladas en el prensachapa.

\section{Entrevistas a comercializadores y consumidores de acero} AISI/SAE 1006 destinado a estampado embutición

El hecho de que los industriales reportaran la problemática antes mencionada y no hubieran identificado una causa para el mismo, sugirió, que su origen tuviera que ver con un bajo nivel de conocimiento de los parámetros que definen la conformabilidad del material de tal manera que en el proceso de compra no se tienen elementos suficientes para establecer requerimientos acertados del material solicitado. Para determinar la validez de esta hipótesis, se realizaron visitas a tres empresas consumidoras de lámina para estampado/embutición ubicadas en el Valle de Aburrá y a dos empresas comercializadoras de este tipo de lámina (una ubicada en Medellín y la otra en Barranquilla), en las que se hicieron preguntas simples tendientes a determinar si las personas entrevistadas tenían conocimiento de o estaban familiarizadas con los parámetros $\mathrm{r}$, rm y $\mathrm{n}$ antes descritos.

\section{RESULTADOS Y ANÁLISIS}

\section{A. Caracterización de materiales}

En la tabla 1 se presentan las composiciones químicas de los aceros analizados junto con los requerimientos del estándar SAE J403 para el acero 1006. En la tabla 2 se muestran las composiciones químicas de aceros al carbono destinados a embutición, de acuerdo con el estándar SAE J2329 [10]. En la tabla 1 se puede ver que los tres aceros analizados cumplen 
tanto los requerimientos de composición química del acero 1006 como los del estándar SAE J2339 grado 1 (especificación con la que actualmente se designa el acero laminado en frio calidad comercial, CRCQ, designación con la que es comúnmente comercializado el acero AISI/SAE 1006 en Colombia ) mostrados en la tabla 2; de hecho, los aceros 1 y 2 cumplen los requisitos de composición química para el acero SAE J2329 grado 4 (conocido anteriormente como CRDDQ) mientras que el acero 3 cumple los requerimientos de composición química del acero SAE J2329 grado 3 (conocido antes como CRDQ). Es importante notar que en los aceros analizados se encontraron titanio y molibdeno, elementos de aleación que junto con el aluminio actúan como refinadores de grano que pueden conducir a aumentos en la resistencia y disminución en la ductilidad [14]. Sin embargo, su efecto definitivo se analizó a partir de los resultados de las pruebas mecánicas y el análisis metalográfico que se discuten a continuación.

\begin{tabular}{ccccc}
\hline $\begin{array}{c}\text { Elemento } \\
\text { (wt\%) }\end{array}$ & $\mathbf{1}$ & $\mathbf{2}$ & $\mathbf{3}$ & $\mathbf{1 0 0 6}$ \\
\hline $\mathrm{C}$ & 0,049 & 0,027 & 0,084 & 0,08 max. \\
$\mathrm{Cr}$ & 0,059 & 0,028 & 0,014 & - \\
$\mathrm{Ni}$ & 0,019 & 0,007 & 0,003 & - \\
$\mathrm{Si}$ & 0,022 & 0,022 & 0,014 & - \\
$\mathrm{Mn}_{\max }$ & 0,149 & 0,209 & 0,238 & 0,45 \\
$\mathrm{Ti}$ & 0,002 & 0,002 & 0,002 & - \\
$\mathrm{P}_{\max }$ & 0,018 & 0,012 & 0,015 & 0,03 \\
$\mathrm{Mo}$ & 0,007 & 0,006 & 0,004 & - \\
$\mathrm{Cu}$ & 0,054 & 0,01 & 0,003 & - \\
$\mathrm{V}$ & - & - & - & - \\
$\mathrm{S}_{\max }$ & 0,006 & 0,016 & 0,005 & 0,05 \\
$\mathrm{Al}$ & 0,053 & 0,04 & 0,028 & - \\
\hline
\end{tabular}

Tabla 1. Composición química de los aceros analizados y el acero AISI/SAE 1006

\begin{tabular}{cccccc}
\hline $\begin{array}{c}\text { Elemento } \\
\text { (wt\%) }\end{array}$ & Grado 1 & Grado 2 & Grado 3 & Grado 4 & Grado 5 \\
\hline C max. & 0,13 & 0,10 & 0,10 & 0,08 & 0,02 \\
Mn max. & 0,60 & 0,50 & 0,50 & 0,40 & 0,30 \\
P max. & 0,035 & 0,035 & 0,030 & 0,025 & 0,025 \\
S max. & 0,035 & 0,030 & 0,030 & 0,025 & 0,025 \\
Al min. & 0,020 & 0,020 & 0,020 & 0,020 & 0,020 \\
\hline
\end{tabular}

Tabla 2. Composición química de los aceros al carbono para estampado de acuerdo con SAE J2329

En lo referente a propiedades mecánicas, los resultados obtenidos para los aceros estudiados se presentan en la tabla 3 mientras que, para efectos de comparación, en la tabla 4 se muestran los requerimientos de acuerdo con SAE J2329. En este caso los tres aceros cumplen los requerimientos del acero SAE J2329 grado 1. Sin embargo, se puede observar que los aceros 2 y 3 cumplen los requerimientos para el grado 2 en el estándar SAE J2329. Más aún, excepto por el valor de rm que es muy cercano al del estándar, se podría decir que el acero 3 satisface los requisitos para el acero SAE J2329 grado 3. Lo anterior ayudaría a explicar el por qué dicho acero, que era el tradicionalmente utilizado por los industriales del país, presenta mejor desempeño en lo referente a conformabilidad comparado con los aceros 1 y 2 . Por lo anterior, se puede decir que el acero 1 corresponde a un acero SAE J2329 grado 1 mientras que los aceros 2 y 3 corresponde a un acero SAE J2329 grado 2.

\begin{tabular}{cccc}
\hline PROPIEDAD & \multicolumn{3}{c}{ ACERO } \\
& $\mathbf{1}$ & $\mathbf{2}$ & $\mathbf{3}$ \\
\hline Resistencia a la & 150,5 & 239,32 & 164,14 \\
Cedencia (Mpa) & & & \\
Resistencia a la & 264,21 & 305,81 & 290,60 \\
Tracción (Mpa) & & 39,10 & 42,02 \\
Elongación (\%) & 32,82 & 1,23 & 1,48 \\
\hline rm & 1,44 & \\
\hline
\end{tabular}

Tabla 3. Propiedades de los aceros analizados

\begin{tabular}{cccccc}
\hline \multirow{2}{*}{ PROPIEDAD } & \multicolumn{5}{c}{ ACERO SAE J2329 } \\
& Grado 1 & Grado 2 & Grado 3 & Grado 4 & Grado 5 \\
\hline $\begin{array}{c}\text { Resistencia a la } \\
\text { Cedencia (Mpa) }\end{array}$ & N/R & $140-260$ & $140-205$ & $140-185$ & $110-170$ \\
$\begin{array}{c}\text { Resistencia a la } \\
\text { Tracción (Mpa) }\end{array}$ & N/R & 270 & 270 & 270 & 270 \\
Elongación (\%) & N/R & 34 & 38 & 40 & 42 \\
Im & N/R & N/R & 1,5 & 1,6 & 1,7 \\
n & N/R & 0,16 & 0,18 & 0,20 & 0,22 \\
\hline
\end{tabular}

Tabla 4. Propiedades mecánicas requeridas de aceros al carbono para estampado de acuerdo con SAE J2329

Finalmente, los resultados del análisis metalográfico (ver figura 3) realizado a los tres aceros estudiados reveló una microestructura con granos alargados como consecuencia del proceso de laminado en frio que es común a los tres aceros. Sin embargo, se hace evidente la diferencia en tamaño de grano entre los aceros estudiados, que coincide con mayores contenidos de elementos refinadores de grano (Al, Ti y Mo) encontrados en los aceros 1 y 2 comparados con la composición química del acero 3 (ver tabla 2). Sin embargo, las diferencias en tamaño de grano también pueden atribuirse a diferencias en el número de ciclos de recocido al que son sometidas las láminas durante el proceso de laminado, hipótesis cuya verificación sobrepasa el alcance de este estudio.

\section{B. Simulación inversa}

Con base en los resultados de la caracterización de materiales, se usaron tomaron los aceros SAE J2329 grado 1 (para el acero 1) y SAE J2329 grado 2 (para los aceros 2 y 3) de la librería de materiales del paquetevAltair Hyperform para realizar la simulación por elementos finitos. Los resultados de la simulación por elementos finitos, figura 4 , corroboraron la aparición de defectos por ruptura o rasgadura (zonas oscuras señaladas con flechas en las esquinas de la pieza embutida) cuando se usan los aceros SAE J2329 grado 1 (figura 4-1) y 
SAE J2329 grado 2 (figura 4-2), resultado que contrasta con la no aparición de defectos en producción cuando se usa el acero 3. Por lo anterior, y tomando en cuenta que lo discutido en la sección de caracterización de materiales (en particular lo cercano del valor de rm obtenido para el acero 3 respecto al requerimiento del acero SAE J2329 grado 3), se decidió realizar la simulación con acero SAE J2329 grado 3 incluido en la librería del paquete Altair Hyperform. Los resultados de esta nueva simulación (ver figura 4-3) corroboran la no aparición de rasgaduras que coincide con las observaciones en producción. Hecho que ya se esperaba a partir de los resultados obtenidos en el análisis metalográfico y los ensayos mecánicos llevados a cabo durante la etapa de caracterización dada la cercanía del valor rm requerido para el acero SAE J2329 grado 3 y el obtenido en la caracterización del acero 3, así como la mayor elongación presentada por dicho acero comparada con el requerimiento de acero SAE J2329 grado 3, la cual, en ausencia de valores de $\mathrm{n}$ y $\mathrm{k}$ experimentales puede ayudar a inferir la conformabilidad de la lámina en operaciones de estamapado/embutición en las cuales predomina el estiramiento de la lámina en una dirección preferencial (algo que se asemeja a las condiciones en las que se realizan las pruebas de tracción).

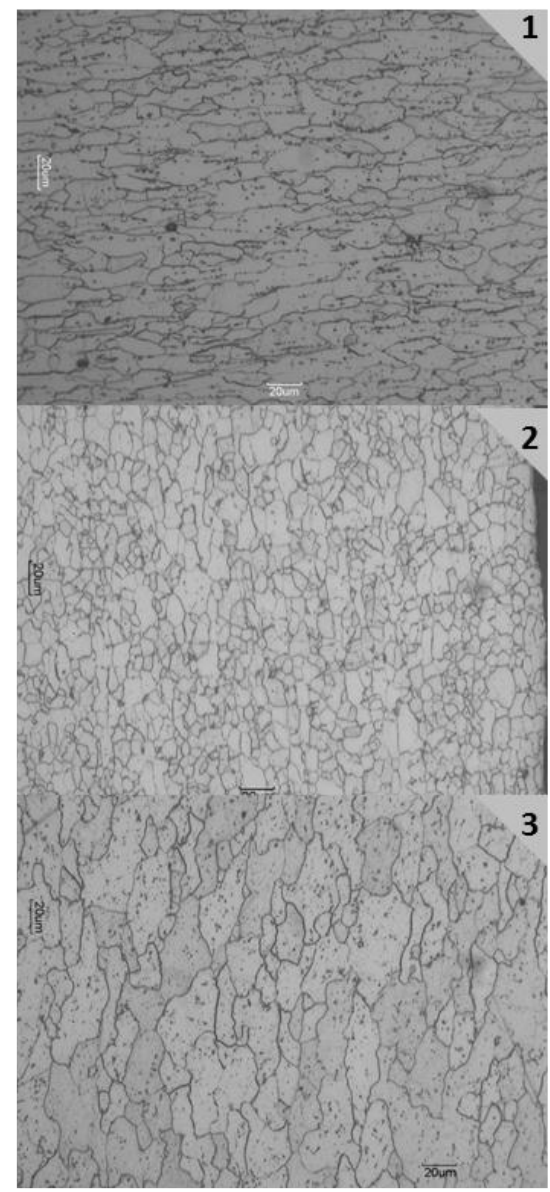

Figura 3. Fotomicrografías de la sección longitudinal (en la dirección de laminación) de los aceros estudiados. Tomadas a 200X y atacadas con nital al $5 \%$

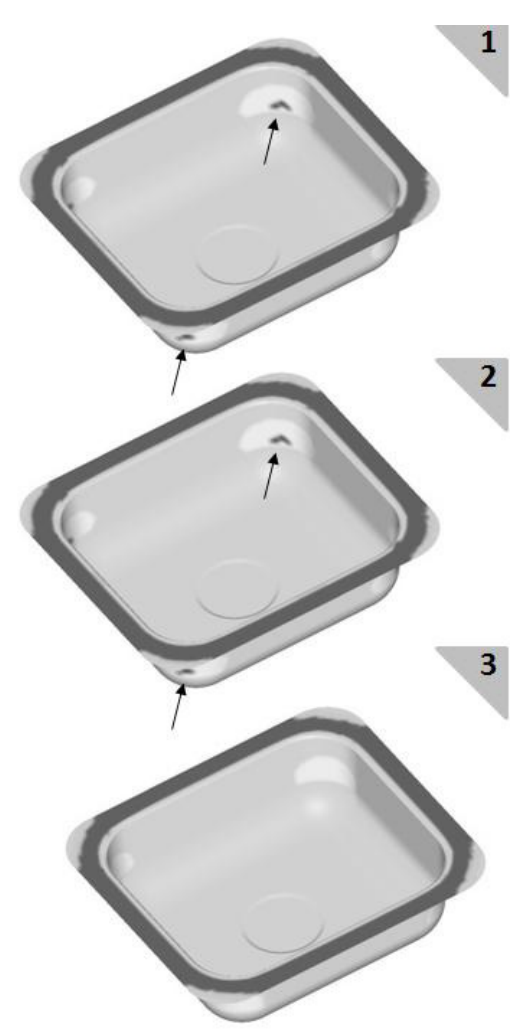

Figura 4. Resultados de conformabilidad obtenidos mediante simulación por elementos finitos para cada uno de los aceros estudiados

\section{Entrevistas a comercializadores y consumidores de acero} AISI/SAE 1006 destinado a estampado embutición

Las entrevistas realizadas a los comercializadores y consumidores de lámina de acero AISI/SAE 1006 mostraron que en todos los casos las personas que atendieron a la entrevista no sólo no estaban familiarizadas con parámetros como el coeficiente de anisotropía (r), el exponente de endurecimiento por deformación (n) y la razón de anisotropía plástica normal $\left(\mathrm{r}_{\mathrm{m}}\right)$; sino que, en la mayoría de los casos manifestaron no conocer su existencia, lo cual conduce a pensar que en la industria nacional la compra de láminas metálicas destinadas a estampado o embutición se hace con base en especificaciones para aceros de uso general, o en algunos casos estructural, que no brindan la información suficiente para inferir el comportamiento del material cuando éste es sometido a operaciones de conformado por deformación plástica como el estampado o la embutición.

Adicionalmente, las entrevistas a los comercializadores de lámina revelaron que la percepción que tienen de su sector se caracteriza por una mayoría que no posee departamento de soporte técnico ni vendedores técnicos que puedan sugerir a los compradores de lámina un tipo de material para una aplicación específica o resolver problemas de calidad asociados con el material. También manifestaron que perciben un considerable nivel de desconocimiento e informalidad en el 
sector de metalistería del país, que se hace más evidente en empresas pequeñas

Las fotografías y figuras deben ser originales, pueden ser en blanco y negro o a color con una resolución de 150 ó 200 dpi. Deben llevar numeración arábiga de acuerdo con su orden de aparición además del nombre en la parte inferior de la figura en letra Times New Roman de 9 puntos.El nombre de la figura debe tener la mejor explicación posible.

\section{CONCLUSIONES}

Los resultados de este estudio muestran que las láminas de acero AISI/SAE1006 que se comercializan en Colombia como lámina conformada en frío calidad comercial (CRCQ, o SAE J2329 grado 1 como se designa actualmente), en algunos casos puede presentar, desde el punto de vista de la conformabilidad, comportamiento similar al de láminas de acero al carbono laminadas en frío calidad embutición (CRDQ, o SAE J2329 grado 3 como se les designa actualmente) dependiendo del proveedor.

Este hecho indujo a la aparición constante de defectos (principalmente rasgaduras) cuando la fluctuación del mercado mundial del acero produjo la llegada al país de nuevos fabricantes de las láminas mencionadas, dado que la lámina de acero AISI/SAE 1006 que se comercializaba regularmente en Colombia correspondía a aquella con características de conformabilidad similares a las del acero SAE J2329 grado 3 (CRDQ).

Lo anterior muestra que la aparición constante defectos reportada por los industriales Colombianos se hubiera podido predecir si los comercializadores y consumidores de chapa destinada a operaciones de estampado o embutición contaran con un conocimiento adecuado de los parámetros que determinan la conformabilidad de dichos materiales cuando son sometidos a los procesos de manufactura mencionados. Sin embargo, en este estudio se encontró que el conocimiento de los actores involucrados con la comercialización y consumo de lámina metálica para estampado/embutición respecto a los parámetros mencionados es deficiente.

Finalmente, los resultados presentados permiten ver que un adecuado conocimiento de los materiales en conjunto con herramientas de simulación por elementos finitos permiten evaluar el efecto que tendría un cambio de material no sólo sobre el proceso de manufactura en general sino sobre la conformabilidad de cada pieza específica

\section{AGRADECIMIENTOS}

Este trabajo fue financiado con recursos del patrimonio autónomo fondo nacional de financiamiento para la ciencia, la tecnología y la innovación Francisco José de Caldas mediante contrato RC-206-2011 y la Universidad Nacional de Colombia Sede Medellín. Los autores también agradecen a Industrias Haceb S.A. por la donación de los materiales y el suministro de los modelos CAD utilizados para llevar a cabo este estudio.

\section{REFERENCIAS}

[1] J. Bringas. Handbook of comparative world steel standards. ASTM International $3^{\text {rd }}$ ed. pp 21-47. Materials Park. USA. 2004.

[2] SAE J403. Chemical Compositions of SAE Carbon Steels. SAE standard. pp 1-10. Warrendale. USA. 2001.

[3] S. Hu, Z. Marciniak, J. Duncan. Mechanics of sheet metal forming. Butterworth-Heinemann Second edition. pp 112. Oxford. England. 2002

[4] ASM International. Metals handbook volume 14 forming and forging. ASM. pp 1930-1942. Materials Park. USA. 2006

[5] W. Hosford, R. Caddell. Metal forming mechanics and metallurgy. Cambridge University press $3^{\text {rd }}$ edition. pp 30-207.Cambridge. England. 2007

[6] D. Banabic, H. Bunge, K. Pöhlandt, A. Tekkaya. Formability of metallic materials. Springer. pp 119-168. Berlin. Germany. 2002

[7] ASTM E646-00. Standard test method for tensile strainhardening exponents ( $\mathrm{n}$-values) of metallic sheet materials. ASTM standard. pp 1-8. West Conshohocken. USA. 2000

[8] ASTM E517-00. Standard test method for plastic strain ratio $r$ for sheet metal. ASTM standard. pp 1-8. West Conshohocken. USA. 2000

[9] ASTM A1008-04. Standard specification for steel, sheet, cold-rolled, carbon, structural, high-strength low-alloy and high-strength low-alloy with improved formability. ASTM standard. pp 1-9. West Conshohocken. USA. 2004

[10] American Iron and Steel Institute \& Auto/Steel Partnership. Automotive steel design manual. American Iron and Steel Institute \& Auto/Steel Partnership. pp 131. Southfield. USA. 2002

[11]B. Tang, J. Sun, Z. Zhao, J. Chen, X. Ruan. "Optimization of drawbead design in sheet forming using one step finite element method coupled with response surface methodology". Int J Adv Manuf Technol. Vol. 31. pp 225-234. 2006

[12] A. Tekkaya. "State-of-the-art of simulation of sheet metal forming". Journal of Materials Processing Technology. Vol. 103. pp. 14-22. 2000

[13]ASTM A370-03. Test Methods and Definitions for Mechanical Testing of Steel Products. ASTM standard. pp 1-11. West Conshohocken. USA. 2003

[14] G. Totten. Steel heat treatment handbook. CRC 2nd ed. p 2-87. Boca Ratón. USA. 2006. 